[Aus der Universitäts-Augenklinik zu Halle a. S.]

\title{
Weitere Untersuchungen über Keratoconus mit dem Abderhaldenschen Dialysierverfahren.
}

\author{
Von \\ Prof. E. v. Hippel \\ in Halle a. $\mathrm{S}$.
}

In meiner ersten Arbeit ${ }^{1}$ ) über diesen Gegenstand bezeichnete ich weitere Untersuchungen als notwendig, um zu erfahren, ob die Störungen der inneren Sekretion, über die ich damals berichten konnte, als ein besonders häufiger oder gar einigermassen regelmässiger Befund zu betrachten seien.

Durch das Entgegenkommen zahlreicher Kollegen bin ich nunmehr in der Lage, im ganzen über 34 serologisch untersuchte Fälle von Keratoconus zu berichten.

Da die Ansicht, dass bei dieser Krankheit eine Störung der inneren Sekretion vorliegt, auf Grund rein klinischer Untersuchungen aufgestellt worden ist, so erscheint es mir richtig, soweit mir Ergebnisse der körperlichen Untersuchungen mitgeteilt worden sind oder soweit ich sie von eigenen Fällen besitze, dieselben im einzelnen aufzuführen.

Zunächst noch einige Angaben aus der Literatur. Meiner Arbeit in den „Klin. Monatsbl." folgte unmittelbar eine das gleiche Thema behandelnde von $\mathrm{Behr^{2 }}$ ) aus der Kieler Klinik. Dieser Autor berichtet über einen Fall, bei welchem gleichzeitig Verdünnung (Blaufärbung) der Sklera im vorderen und myopische Dehnung im hinteren Bulbusabschnitt, ausserdem habituelle Luxationen in verschiedenen Gelenken vorhanden waren.

Er zieht daraus den Schluss, dass eine kongenital bedingte

1) E. v. Hippel, Zur Ätiologie des Keratoconus. Klin. Monatsbl. f. Augenheilk. Bd. LI, 2. B. 273.

2) Behr, Beitrag zur Ätiologie des Keratoconus. Ibid. S. 281. 
Entwicklungsstörung die wahrscheinlichste Ursache des Keratoconus sei.

Die von Siegrist für das Vorhandensein eines Hypothyreoidismus angeführten Symptome fehlten in diesem Falle. Auch in einem zweiten, der einen kräftigen Mann betraf, wurden sie vermisst.

Behr lässt die Möglichkeit offen, dass es zwei Arten des Keratoconus gibt, die eine bedingt durch kongenitale Anlage als Entwicklungsstörung, die andere erworben auf der Basis eines dyskrasischen Zustandes.

Groth ${ }^{1}$ ) (Dissertation aus der Petersschen Klinik) betont, dass es sich beim Keratoconus wenigstens in einer Anzahl von Fällen um eine kongenital angelegte Bildungsanomalie handle, lässt aber wenigstens die Möglichkeit offen, dass Störungen der inneren Sekretion gelegentlich mitspielen könnten.

Mit der Abderhaldenschen Methode haben $\mathrm{Hack}^{2}$ ) and Tamascheff ${ }^{3}$ Fälle von Keratoconus untersucht.

Hack berichtet über 3 Fälle. Im ersten reagierten Basedowstruma und Gehirn positiv, Struma, Nebenniere und Karzinom negativ. 5 Wochen später zeigten Schilddrïse, Basedowkropf, Kropf, Thymus, Ovarium, Placenta und Gehirn keinen Abbau. Die positive Reaktion mit Gehirn bei der ersten Untersuchung erscheint mir mindestens sehr auffallend, da ein Grund für dieselbe nicht ersichtlich ist.

Der zweite völlig negativ reagierende Fall erscheint mir hinsichtlich der Diagnose zweifelhaft, da bei positivem Wassermann auf beiden Augen ausgedehnte parenchymatöse Hornhauttrübungen vorhanden waren und die Ektasie der Cornea oberhalb der Mitte sass.

Im dritten Falle gibt Hack an: positive Reaktion mit Thymus, negative mit Sehilddrüse, Kropf, Basedowkropf, Placenta und Ovarium.

Tamascheff veröffentlicht 2 Fälle:

1. 19jähriges Mädehen mit dentlichen Zeichen von „Dysthyreoidismus" (vergrösserte und verhärtete Schilddrüse, Tremor, Tachykardie, Lymphozytose, erhöhte Blutgerinnbarkeit, positiver Abderhalden, leider ist aus dem Referat nicht ersichtlich, mit welchen Organen positive Reaktion erhalten wurde. Nach innerlichem Gebrauch von Schilddrüsentabletten and subkutanen Arseninjektionen (innerhalb eines Monats) Abflachung des Keratoconus und Besserung des Visus von Finger $1 \mathrm{~m}$ auf Finger $3 \mathrm{~m} \mathrm{bzw}$. von 0,1 auf 0,3 bis 0,4 .

1) Groth, Zur Ätiologie des Keratoconus. Diss. Rostock 1913.

2) Hack, Zur Ätiologie des Keratoconus. Arch. f. Augenheilk. Bd. LXXVI. S. 259.

3) Tamascheff, Zur Frage der Ätiologie und Therapie des Keratoconus. Russki Wratsch. XIII. Nr. 4. Ref. im Zentralbl. f. d. ges. Ophth. Bd. I. S. 185. 
2. 26 jährige $\mathrm{Zweitgebärende} \mathrm{mit} \mathrm{linksseitigem} \mathrm{typischem} \mathrm{Keratoconus,}$ daneben Zeichen von Dysthyreoidismus. (Tachycardie, Anämie, positive Abderhaldensche Reaktion; womit?) Nach Thyreoidinanwendung nach einem Monat Besserung des Visus von 0,1 auf 0,5 .

Tschirkowsky ${ }^{1}$ ) bemerkte in der Diskussion zu dem Vortrag Tamascheffs, dass er einen Fall von Keratoconus mit gleichzeitiger Sklerodermie kenne, die ja auch mit Störungen der inneren Sekretion zusammengebracht werde.

Sonstige einschlägige Mitteilungen habe ich nicht gefunden.

Von eigenen Fällen, die ich seit meiner früheren Mitteilung gesehen habe, sind folgende zu erwähnen:

1. Franz Singer, 8 Jahre, beiderseits Keratoconus $S=53_{35}$. Hornhautsensibilität normal, kein brauner Ring vorhanden. Ziemlich ausgedehnte zentrale Hornhauttrübung mit unregelmässigem Astigmatismus, Gläser bessern nicht.

Die Allgemeinuntersuchung ergab keinen pathologischen Befund, sie war aber nicht mit der gleichen Genauigkeit ausgeführt worden, wie in anderen Fällen. Der Versuch, sie zu ergänzen, scheiterte an der Verständnislosigkeit der Eltern des kleinen Patienten.

8.9. X. 1913. Abderhalden, $\mathbf{1 , 5} \mathrm{ccm}$.

$$
\begin{array}{ll}
\text { Ser. - Thyr. } & \\
" \text { + Thy } & \oplus \\
"+\text { Thymus } & \oplus \\
" \text { + Nebenniere } & - \\
" \text { + Pankreas } & \oplus \\
"+\text { + Milz } & -
\end{array}
$$

2. Wilhelm Wischeropp, 16 Jahre. Seit 2 Jahren Abnahme des Sehvermögens rechts. Typischer Keratoconus. $-18 D$ sph. $\bigcirc-7 D$ eyl. A vert. $S=\left.{ }^{b}\right|_{20}$. Links $-2 D S=\left.{ }^{5}\right|_{4}$. Minimale Trübung an der Kegelspitze. Temporal ein winziges graues Pünktchen in der Linse. Sensibilität an der Kegelspitze ein wenig herabgesetzt. Nach zweimaliger Kauterisation $-4 \leftrightharpoons-1$ cyl. av. $S={ }^{5} / 15$.

Allgemeinbefund (Prof. Mohr). Klein, mager, Skoliose der Brustwirbelsäule, Lordose der Lendenwirbelsäule, Residuen alter Rhachitis am Brustkorb und den Extremitäten. Genitale normal. Tonsillen hyperplastiseh, verdiekte aufgeworfene Ober- und Unterlippen, adenoider Gesichtsausdruck. Zungenspitze geschwollen. Speicheldrüsen normal. Schilddrüse: Isthmus fühlbar, aber nicht pathologiseh. Im Jugulum fühlt man bei starkem Pressen eine weiche Masse oberhalb des A ortenbogens. Keine Sternaldämpfung. Herz, Lungen ohne Besonderheiten.

1) Ibid.

$$
\begin{aligned}
& \text { Hämoglobin } \quad 75 \% \\
& \text { Leukozyten }
\end{aligned}
$$




$$
\begin{aligned}
& \begin{array}{lr}
\text { neutroph. } & 42 \% \\
\text { eosinoph. } & 4 \%
\end{array} \\
& \text { grosse Lymphoz. } 32 \% \\
& \text { kleine } \quad, \quad 16 \% \\
& \text { Mastzellen } 6 \%
\end{aligned}
$$

Gerinnungszeit nach Wright $\left.3^{1}\right|_{2} ^{\prime}$ (normal $2-6^{\prime}$ ).

Röntgendurchleuchtung: Oberes Mediastinum frei, Herz median gestellt, Kugelherz.

Diagnose: Status lymphaticus.

29./30. I. 1914. Abderhalden (1,0 ccm):

$$
\begin{gathered}
\text { Ser. - } \\
" \text { + Thyr. } \\
" \text { + Thym. } \\
" \text { + Nebenniere - } \\
" \text { + Pankreas - } \\
" \text { + Hoden - } \\
" \text { + Herz } \\
" \text { + Milz } \\
" \text { + Niere } \\
" \text { + Leber }
\end{gathered}
$$

26.|27. V. 1914. Untersuchung wiederholt, $1,5 \mathrm{ccm}$.

$$
\begin{aligned}
& \text { Ser. - } \\
& " \text { + Thyr. } \\
& " \text { + Thym. - } \\
& " \text { + Nebenniere - } \\
& "+\text { Pankreas - } \\
& " \text { + Hoden - }
\end{aligned}
$$

3. Ernst Günther, 25 Jahre, Journ.-Nr. 1049. jektion.

Angeblich blind geboren. Lichtschein für kleinste Lampe, falsche Pro-

Beiderseits typischer Keratoconus mit Trübung der Spitze. Wann der Keratoconus entstanden ist, unbekannt. Im poliklinischen Journal 1903 nicht erwähnt. Beiderseits gelbliche Verfärbung der Papillen, ausgedehnte Retinalatrophie mit sehr starker Pigmentierung, Nystagmus, Wassermann -.

Allgemeinuntersuchung (Prof. Mohr). Kräftiger Mann mit genügendem Fettpolster, brachykephaler Schädel. Beide Hoden abnorm klein, Penis von normaler Grösse, Behaarung von maskulinem Typus. Vasomotorische Übererregbarkeit.

Im Jugulum fühlt man deutlich den gleichmässig verdickten Isthmus der Schilddrüse, rechter Lappen deutlicher zu fühlen als linker. Keine Drüsenschwellungen, keine Sternaldämpfung. Lungen ohne Besonderheiten, Herzstoss hebend, im IV. Interkostalraum, nach rechts verbreitert. Voussure. Systolisches Schwirren. Systolisches Geräusch an der Spitze und an der Aorta. Puls im Verhältnis zum Spitzenstoss klein. Milz, Leber ohne Besonderheiten.

$$
\begin{array}{lr}
\text { Hämoglobin } & 98 \% \\
\text { Leukozyten } & 9600
\end{array}
$$


Weitere Untersuch. über Keratoconus mit dem Abderhaldenschen usw. 177

$\begin{array}{lr}\text { Neutroph. } & 66 \% \\ \text { Lymphoz. } & 29 \% \\ \text { Eosin. } & 4 \% \\ \text { Mastz. } & 1 \%\end{array}$

Röntgenaufnahme des Schädels: Tiefe Einsenkung der Basis, kleine mittlere Schädelgrube. Sella ohne Befund. Hoher senkrechter Durchmesser, ungleiche Dicke der Schädelkapsel. Diagnose: Herzhypertrophie, verdickter Isthmus der Schilddrüse, abnorm kleine Hoden.

Abderhalden (1 cem) Ser. -

$\begin{array}{ll}"+\text { + Thyr. } & + \\ "+\text { Thymus } & \oplus \\ "+\text { + Nebenniere } & - \\ "+\text { + Pankreas } & - \\ " \quad+\text { Hoden } & \oplus \\ " \quad+\text { Milz } & - \\ " \quad+\text { Niere } & -\end{array}$

Es darf besonders hervorgehoben werden, dass die klinische und serologische Untersuchung gemacht wurde, ohne dass die beiderseitigen Ergebnisse bekannt waren.

4. Frau Juliane Bräuer, 39 Jahre, Journ.-Nr. 1098.

Sieht schon seit dem 6. Lebensjahr schlecht, damals sollen schon Linsentrübungen vorhanden gewesen sein. In der Familie kein Augenleiden. Patientin fühlt sich sonst wohl, sebwitzt nur sehr viel, die Haare gehen ihr ziemlich stark aus.

Beiderseits Keratoconus mit starker Trübung der mittleren Hornhautpartien, die Trübung reicht aber fast bis an den oberen Rand, keine Gefässe. Rechts Subluxatio Lentis nach aussen, Linsenrand sichtbar. Myop. Fundus. Links Iridodonesis, totale Katarakt. Linsenrand nicht zu sehen, Subluxatio vermutet.

Bei der Operation des linken Auges sofort Glaskörpervorfall und nach Extraktion der Linse mit Schlinge retrochoroideale Blutung.

Allgemeinuntersuchung ( Prof. Mo hr). Kopfschmerzen, auffallendes Schlechterwerden der Zähne seit 2 Jahren. Drüsenschwellangen am Hals und in der Leiste, Tonsillen gross, leicht gerötet. Thyreoidea palpabel, weich, Isthmus etwas härter. Herz systolisches Geräusch, sonst normaler Organbefund.

$\begin{array}{lr}\text { Hämogl. } & 85 \% \\ \text { Erythroz. } & 3664000 \\ \text { Leukoz. } & 7600 \\ \text { eosinoph. } & 1 \% \\ \text { neutroph. } & 66 \% \\ \text { grosse Lymphoz. } & 14 \% \\ \text { kleine }, & 13 \% \\ \text { mononukl. } & 2 \% \\ \text { Überg. Formen } & 3 \% \\ \text { Mastzellen } & 1 \%\end{array}$


Also wesentlich normaler Befund. Abderhalden, $1 \mathrm{ccm}$ :

$$
\begin{aligned}
& \text { Ser. - } \\
& " \text { + Thyr. } \\
& "+\text { + Thym. - } \\
& " \text { + Thym. - } \\
& " \text { + Mvar. } \\
& " \text { + Leber - }
\end{aligned}
$$

Von den Fleischersehen Fälen sind in meiner fruheren Arbeit die serologischen Befunde zu Nr. 1, 4, 5, 6 angegeben. Später wurden noch untersucht Fall 2, 9 und 11. Nr. 11 muss ausscheiden, da Serum allein schon enorm stark positiv reagierte, das Serum sah auch rötlich aus, jedenfalls hämolytisch:

Fall $2 \mathrm{gab}$ folgenden Befund (1,5 cem):

Ser. -

Fall 9. 1,5 cem: Ser. $\oplus$
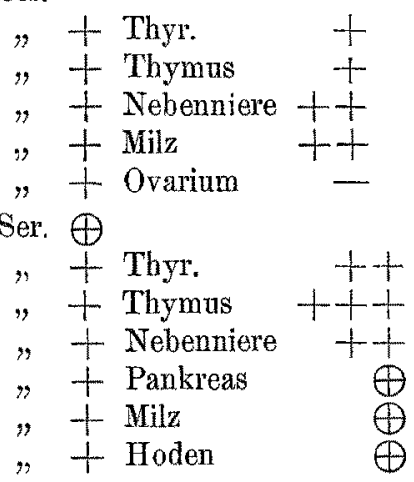

also Thyreoidea, Thymus und Nebenniere positiv. Auf die klinischen Befunde der Fleischerschen Fälle verweise ich und führe sie hier nicht nochmals an.

Zu dem Axenfeldschen Falle, dessen serologischen Befund ich hier noch wiederhole, liegen mir folgende klinischen Notizen vor:

32 jährige Patientin, seit früher Jugend chronisches universelles Ekzem, erst "Milchschorf", đann Ausschlag an den Gelenken und Bänden, mit 11 Jahren schwere Masern, mit 13 Jahren Bauehfelltuberkulose und eitrige Halsdrüsen. Operationen. Dann Typhus, ${ }^{1 / 2} \mathrm{Jahr}$ krank. Hautleiden fast ganz verschwunden, kam dann aber verstärkt wieder. Magenkrämpfe, Gallensteinkoliken, dysmenorrhoische Beschwerden. Kur in Elster mit schlechtem Erfolg für die Haut, 1906 Röntgenbehandlung mit schweren Hantrerbrennungen. Später Blinddarmreizung, Gelbsucht, Diphtherie, Rippenfellentzündung. In den letzten Jahren wesentliche Verschlimmerung des Unterleibsleidens.

Der Keratoconus ist auf der einen Seite schon seit Jahren höchstgradig (pulsierend), auf dem andern noch lesefähigen A uge ununterbrochen progressiv. Zur Zeit der Abderhaldenschen Untersuchung war wieder eine deutliche Zunahme aufgetreten. 
Weitere Untersuch. über Keratoconas mit dem Abderhaldenschen usw. 179

Abderhalden. 1,5 ccm: Ser. $\oplus$

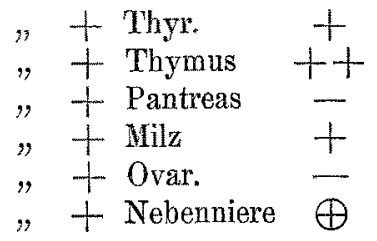

Dieser Fall ist in der ersten Zeit unserer Beschäftigung mit der $A b$ derhaldenschen Methode von Igersheimer untersucht worden. Es fällt auf, dass bei Serum $\oplus$ einige Organe negativ reagiert haben.

$\mathrm{Zu}$ dem bereits früher serologiseh untersuchten Fall Augsteins ist die briefliche Mitteilung des Autors von Interesse, dass der Patient bei Weglassen des Thyradens wieder Epitheltrübungen bekommen hat, die bei Wiederaufuahme der Therapie prompt verschwanden. Die Struma ist nicht wiedergekehrt, ebensowenig die trophischen Störungen an den Nägeln. Nach einem weiteren Bericht Augsteins vom Mai 1914 hat Patient bis Mitte Dezember 1913 im ganzen 1380 Tabletten Thyraden und 1100 Arsenpillen genommen, seitdem keine Medikation mehr. Er fiihlt sich wohl, hat ${ }_{2} / 3$ Sehsehärfe, in den letzten Monaten allerdings wieder einzelne Epitheltrübungen.

Am 19.|20. V. 1914 erneute Untersuchung des Serums:

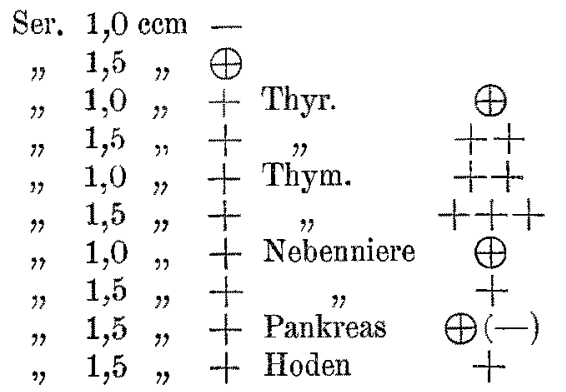

Das Resultat der früberen Untersuchung 18./19. VIII. 1913 lantete $(1,5 \mathrm{ccm})$ :

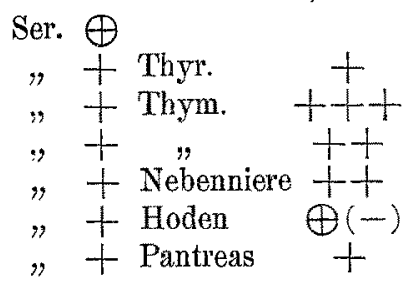

also fast identisches Resultat.

Von Angstein wurden mir noch zwei weitere Sera geschickt, die zu folgenden Füllen gehören.

1. 25jähriger Patient. Doppelseitiger Keratoconus, wahrscheinlich seit Geburt bestehend, kombiniert mit Nystagmus und Sehnervenatrophie. Visus Handbewegungen. Bei einer Schwester soll dasselbe Leiden vorhanden sein. 
Keine körperliche Anomalie, speziell keine Struma. Eine zytologische Blutantersuchung wurde nicht gemaeht.

Abderhalden $(1,5 \mathrm{ccm})$ : Ser. -

$"$ + Thyr. -
$"$ + Thym. -
$"$ + Nebenniere -
$"$ + Hoden -
$"+$ Milz
$"$ + Niere -
$"$ + Leber -

2. 48 jähriger Patient. Seit 12 Jahren Sehvermögen schlechter geworden. Links ausgeprägter Keratoconus. Dicht unter dem Epithel der Spitze zwei langgezogene parallele Trübungen in den vorderen Lamellen, machen den Eindruck eines völlig abgelaufenen Prozesses. Rechts kaum merkliche Vorwölbung im unteren Drittel der Hornhaut, aber $6 \mathrm{D}$ as., mit Korrekt. $5 / 10$.

Allgemeinbefund: Kachektiseher Mann mit eingefallenen Wangen, spärlichem Haar, spröder trockener Haut, dystrophischem Nagel an der 3. linken Zehe. Keine Struma. Urin ohne Besonderheiten, alte Spitzendämpfung rechts. Blutuntersuchung nach Siegrists Angaben negativ, vielfache interne Untersuchung gibt keinen Anhaltspunkt zur Erklärung des enormen Kräfteverfalls. Der Keratoconus hat sich bei der Thyradenbehandlung gebessert.

9./10. II. 1914. Abderhalden (1 cem):

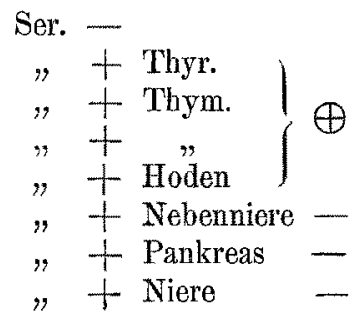

Nochmalige Untersuchung, 9.110. V. 1914, nachdem Patient. 300 Tabletten Thyraden und 300 Arsenpillen genommen thatte (bis unmittelbar vor der Blutentnahme): Ser. -

$$
\begin{aligned}
& " \text { + Thyr. - } \\
& " \text { + Thym. - } \\
& " \text { + Nobenniere - } \\
& " \text { + Nebreas - } \\
& " \text { + Hoden - }
\end{aligned}
$$

Es folgen 2 Fälle von Siegrist, der mir besonders genaue Krankengeschichten zur Verfügung gestellt hat, die ich mit einigen Kürzungen wiedergebe.

1. Geissler, 17 Jahre. Linkes Auge seit 4 Jahren, rechtes Auge seit 2 Jahren erkrankt.

15. XI. 1912 stärkerer Astigmatismus festgestellt, aber noch kein Keratoconus. Dieser wurde erst 25. XI. 1913 am linken Auge gefunden.

Anamnese. In der Familie keine Augenkrankheiten, Mutter äusserst 
Weitere Untersuch. über Keratoconus mit dem Abderhaldenschen usw. 181

nervös. Patient sonst gesund, aber ebenso wie seine Mutter sehr nervös und reizbar, er meint, dies babe noch zugenommen. Eigentümliches Knacken in den Ohren, sobald er erregt ist.

Allgemeinstatus: Gesund aussehender Mann, Haut nicht trocken, Appetit and Verdauung gut. Am Hals einige Drüsen. Thyreoidea nicht vergrössert. Thorax von normaler Wölbung. Lungen und Herz ohne Besonderheiten.

Urin Eiweiss in Spuren, kein Zucker, Diazoreaktion negativ. Indikan vermehrt, Urobilin nicht vermehrt. Blut negativ. Chloride kompakt.

Mikroskopisch normal.

Wassermann negativ.

Untersuchung der Thyreoidea und des Halses auf der Kocherschen Klinik: Schildarüse eher kleiner als normal, keine Thymusdämpfung.

Gehör, Nase, Rachen normal.

Blutuntersuchung (Dr. Kottmann). Gerimnungszeit normal (Beginn nach 17 Minuten, Ende nach 22 Minuten).

$\begin{array}{lrr}\text { Hämogl. } & 90 & \% \\ \text { Erythroz. } & 4853000 & \\ \text { Leukoz. } & 7400 & \\ \text { neutroph. } & 60 & \% \\ \text { Lymphoz. } & 32 & \% \\ \text { eosin. } & 2 & \% \\ \text { Überg, i. grosse mononukl. } & 6 & 0 \% \\ \text { Mastzellen } & 0,1 \%\end{array}$

Blutdruck 125, keine Schilddrüsensymptome, keine Kropfheredität, gutes Gedächtnis, kein Haarausfall, kein Zittern, keine Diarrhoe, keine Abmagerung.

Blutuntersuchungen durch die Augenklinik sowie durch die medizinische Klinik geben den erwähnten so ähnliche Werte, dass sie hier nicht wiedergegeben $\mathrm{zu}$ werden brauchen.

Abderhalden, 11./2. XII. 1913. 1,5 cem:

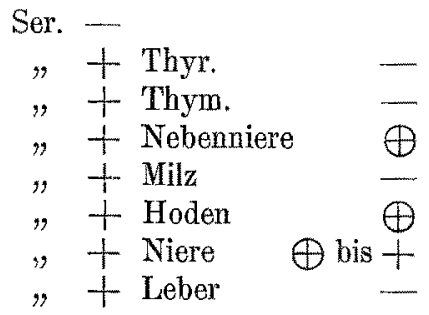

Die positive Reaktion mit Niere war mir deshalb verdächtig, weil ich bei einer jetzt sehr grossen Zahl anderer Untersuehungen Niere ausmahmslos negativ reagierend fand. Er enthält aber Eiweiss.

2. Howald, weiblich, 16 Jahre. Mutter gemütskrank, von zehn Geschwistern eines mit 4 Wochen an Diphtherie gestorben, eines mit 21 Jahren an Tumor der hinteren Schädelgrube, eine Schwester gemütskrank. Patientin selbst erst 5 mal menstruiert, Periode unregelmässig und schwach. Voriges Jahr Ausschlag in den Achselhöhlen und Kniekehlen. Im Alter von 15 Jahren vielHerzklopfen und Schlaflosigkeit. Im Winter 1912/13 Beginn einer Sehstörung. 
7. XI. 1913. Links typischer stark vorgetriebener Keratoconus mit klarer Spitze. Rechts beginnender Keratoconus.

Allgemein befund (Priv.Doz. Dr. Deucher). Guter Ernährungszustand. Lungen normal. Puls im Liegen 96, im Sitzen 102, im Stehen 106. Spitzenstoss hebend, ausserhalb der Mammillarlinie. Herz: links $1 \mathrm{~cm}$ ausserhalb der Mammillarlinie, rechts keine Dämpfung neben dem Sternum. An der Spitze leichtes systolisches Geräusch, an der Pulmonalis systolisches Geräusch, am lautesten über dem Sternum. Aorta leichtes systolisehes Geräusch.

Pulskurve normal, ausser grosser Labilität.

Blutdruek maximal 134, minimal 111.

Nervensystem, Abdominalorgane ohne Besonderbeiten. Wassermann negativ. Pirquet positiv.

Urin: sauer, spez. Gewicht 1024, Eiweiss in ganz geringer Menge. Kein Zucker, Indikan nicht vermelrt. Urobilin vermehrt. Diazoreaktion negativ, Blut negativ, Chloride kompakt, Gallenfarbstoff negativ. Mitrosk.: Leukozyten und Blasenepithelien (Dozent Dr. Wildholz).

Halsorgane: Hals deutlich verdickt. Larynx und Trachea ganz median. Trachea in ihrem Teile über dem Jugulum seitlich komprimiert, so dass Säbelscheidenform, wenn auch nicht sehr ausgesprochen, zustande kommt.

Beide Schilddrüsenlappen deutlich vergrössert, auf das doppelte einer normalen Schilddrüse. Im linken Lappen ein kleinpflaumengrosser Kolloidknoten nahe dem Unterhorn, das übrige Gewebe rein hypertrophisch.

A. thyr. sup. dentlich fühlbar, ohne Schwirren. Drüsensubstanz ohne Pulsation. Deutlicher Isthmus fühlbar. Rechter Schilddrüsenlappen etwas grösser als der linke. In ihm 2 Kolloidknoten.

Blutuntersuchung (Dozent Dr. Kottmann):

$\begin{array}{lrr}\text { Hämogl. } & 70 \% \\ \text { Erythroz. } & 4563000 & \\ \text { Leukoz. } & 7200 & \\ \text { Neutroph. } & 51 & \% \\ \text { Lymphoz. } & 38 \% \\ \text { Eosinoph. } & 5 \% \% \\ \text { Überg. n. Monon. } & 6 \% \% \\ \text { Mastzellen } & 0,6 \%\end{array}$

Abderhalden, $1,5 \mathrm{ccm}$ : Ser. -

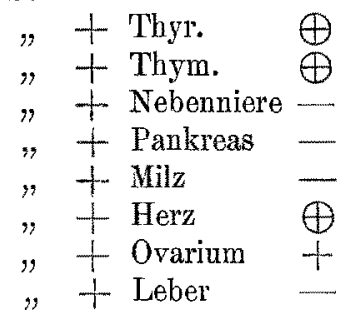

Göttinger Klinik (A. v. Hippel).

1. 19 jähriges Mädchen (Lilii Walter), im 10. nnd 12. Lebensjahre Gelenkrheumatismus mit Endokarditis. Seit 2 Jahren Abnahme des Sehvermögens 
Weitere Untersuch. über Keratoconus mit dem Abderhaldenschen usw. 183

Beiderseits Keratoconus etwas nach aussen und unten. Beiderseits Myop. $5 D$ R. $S=0,3$, L. Finger 4 M. Therapie Kauterisation.

Allgemeinbefund: Kompensierte Aorteninsuffizienz.

$\begin{array}{lr}\text { Hämogl. } & 48 \% \\ \text { Erythroz, } & 5200000 \\ \text { Leukoz. } & 11600 \\ \text { Neutroph. } & 61,5 \% \\ \text { Eosin. } & 1,5 \% \\ \text { Kleine Lymph. } & 28 \% \\ \text { Grosse } " & 9 \%\end{array}$

Abderhalden, 1,5 cem: Ser. -

$$
"+\text { Thyr. }-
$$

2. Frida Grote, 16 Jahre. Seit 1 Jahr Sehstörung. Parazentral unten aussen starker Keratoconus, Spitze leicht grau getrübt. Fundus normal.

Allgemeinbefund (Prof. Lichtwitz): An Thyreoidea und Thymus kein Befund zu erheben. Lungen gesund, Herz von normaler Grösse. Lautes systolisches Geräusch, II. Pulmonalton verstärkt. Nervensystem olne Besonderheiten. Urin ohne Besonderheiten.

$\begin{array}{lr}\text { Hämogl. } & 66 \% \\ \text { Erythroz. } & 5120000 \\ \text { Leukoz. } & 9900 \\ \text { Neutroph. } & 70,8 \% \\ \text { Eosin. } & 2,9 \% \\ \text { Kleine Lymph. } & 19,6 \% \\ \text { Grosse " } & 6,2 \% \\ \text { Mastzellen } & 0,5 \%\end{array}$

Gerinnungszeit nicht bestimmt.

Abderhalden (1,5 ccm):

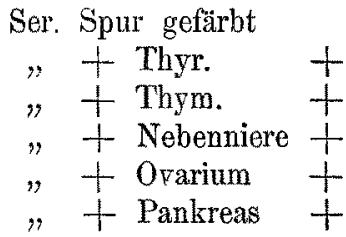

Wagenmann. Heidelberger Klinik (Patient Buchberger).

Rechtes Auge soll seit dem 4. Lebensjahre schlecht sehen, linkes Auge sieht auch schlecht, unbekannt wie lange.

Beiderseits Keratoconus: R. $-7 \mathrm{sph} . \bigcirc-3 \mathrm{eyl} \mathrm{A.} 30^{\circ}$ nas. $S=5{ }_{20}$, L. $-3 D S=5 / 15$.

Allgemeinbefund konnte nicht erhalten werden, da Patient seinen Wohnort wechselte.

Abderhalden 1,5 cem: Ser. -

$$
n+\text { Thyreoid. }
$$




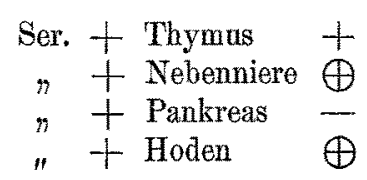

Scheffels-Krefeld, 2 Fälle.

1. 19 jähriger Mann, seit 3 Jahren Sehstörung.

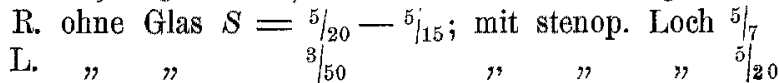

Beiderseits "Keratoconus, Kegelspitze nicht getrübt, an der Descemet mit Hornhautmikroskop, nichts Pathologisehes.

Patient ist nervös, blass, hat aber keine trockene Haut, keine besondere Schweissbildung, keinen Haarausfall und keine Gedächtnisschwäche, keine Heredität.

Abderhalden (1,5 cem): Ser. -

$$
\begin{aligned}
& "+\text { Thyreoid } \\
& "+\text { Thymus }++ \\
& "+\text { Nebenniere } \oplus \\
& "+\text { Pankreas } \oplus
\end{aligned}
$$

2. 42jährige Patientin, seit 5 Jahren beginnender Keratoconus. Seit ungefähr 20 Jahren steht Patientin wegen einer auf hereditärer Lues beruhenden Retinalatrophie mit Pigmentierung in Behandlung. Vielfach mit $H g . K J$. und Salvarsan behandelt.

„Irgendwelche Störungen seitens der Schilddrüse fehlen", Blutdruck 115. Blutbefund :

$$
\begin{array}{lr}
\text { Hämogl. } & 60 \% \\
\text { Erythroz. } & 4800000 \\
\text { Leukoz. } & 3900 \\
\text { Neutroph. } & 48 \% \\
\text { Lymphoz. } & 50 \% \\
\text { Eosinoph. } & 2 \%
\end{array}
$$

Blutplättchen sebr vermindert, Gerinnung verzögert.

Serum (ist ausgesprochen hämolytisch, wird trotzdem angesetzt, das Ergebnis spricht mit Wahrscheinlichkeit dafür, dass die Hämolyse in diesem Falle keinen Einfluss auf die Reaktion gehabt hat).

$$
\begin{aligned}
& \text { Ser. - } \\
& " \text { + Thyr. } \\
& " \text { + Thym. } \\
& " \text { + Nebenniere }- \\
& " \quad+\text { Pankreas - } \\
& "+\text { Milz }
\end{aligned}
$$

Fall von Brenske-Hannover.

27jähriges Fräulein, seit 4. X. 1912 in Behandlung. Schwächliche, "etwas wunderliche" Dame. Haut grau, etwas welk und trocken, an Haaren und Zähnen nichts Besonderes, Vergrösserung der Thyreoidea nicht nachzuaveisen. 
Weitere Untersuch. über Keratoconus mit dem Abderhaldenschen usw. 185

R. ausgesprochener Keratoconus ohne Tribung.

-5 sph. $\bigcirc-6$ eyl. $A v . S=3 / 36^{\circ}$

L. Andeutung von Keratoconus

$$
-3,5=-1,5 \text { cyl. An. } S=\zeta_{16} \text {. }
$$

1 Jahr später: rechts ziemlich unverändert, links deutlicher, Astigmatismus anf $3,5 D$ gestiegen.

Allgemeinbefund fehlt.

Abderhalden.

$$
\begin{aligned}
& \text { Ser. - } \\
& "+\text { Thyr. } \\
& "+\text { Thym. } \\
& "+\text { + Nebenniere - } \\
& "+\text { Pankreas - } \\
& "+\text { - Milz - }
\end{aligned}
$$

Reis-Lemberg. 1 Fall:

R. $S .=\left.5\right|_{50}$, L. $\left.{ }^{1}\right|_{60}$ Gl. b. n. R. zarte, L. dichte Trübung der Kegelspitze. Blutuntersuchung neutroph. $75 \%$, eos. $5 \%$ kleine Ly. $15 \%$, grosse $3,5^{0}$ \% , Uberg. f. $1 \%$. Nach Injektion von 0,01 Pilocarp: neutroph. $67 \%$, eos. $8 \%$ kl. Ly. $14 \%$ grosse $5 \%$ Überg. f. $6 \%$.

Nach 0,01 Atropin, neutr. $57 \%$, eos., $9 \%$, kl. Ly. 23, grosse $8 \%$ Überg. $2 \%$. Nach Adrenalin $1 \mathrm{cem}(1: 1000)$, nentr. $73 \%$, eos. $7 \%$, Mono $3 \%$ Lymphoc. $16 \%$ Uberg. $1 \%$.

Abderhalden (1,5 cem.) Ser. -

\section{Koll-Elberfeld.}

$$
\begin{aligned}
& \text { + + Thyr. - } \\
& " \text { + Thymus - } \\
& " \text { + Nebenniere - } \\
& " \text { + Pankreas - } \\
& " \text { + Hoden - }
\end{aligned}
$$

Weiblicher Patient. Seit einer Reihe von Jahren Keratoconus. In den Entwicklungsjahren Struma, 2 Sohwestern haben sie ebenfalls gehabt. Eine ist operiert worden, bei der andern, sowie bei der Patientin ist die Struma auf Jod zurückgegangen.

Allgemeinbefund (Dr. Koll, Oberarzt der inneren Abteilung Barmen): Schleimbäute blass, Sehilddrüse nicht zu fühlen, Gegend der Schildarüse nicht empfindlich, keine abnorme Pulsation der Halsgefässe. Patientin klagt über Sensationen in der vorderen Halsgegend, die wohl auf die geschwollene Zungenmandel zurückzuführen sind. Lungen, Herz, Abdomen normal. Auch nicht andeutungsweise Zeichon, die auf eine Störung der Schilddrüse hinweisen würden

Hämoglob. $58 \%$, neutrophile $58 \%$, darunter $3 \%$ eosinophile und $1 \%$ Mastzellen. $37,5 \%$ kleine und $3,5 \%$ grosse Lymphozyten, also deutliche Lymphozytose. Keine morphologischen Veränderungen. Achylia gastrica.

Abderhalden $(1,5 \mathrm{ccm})$. Ser. -

$$
"+\text { Thyr. }
$$


Engelbrecht-Erfurt.

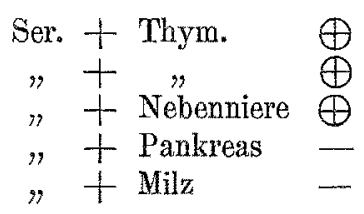

18 jähriger Mann. Vor 3 Jahren an Osteomyelitis krank, seit dieser Zeit Fleck auf der linken Cornea bemerkt, der allmählich grösser wurde. Gleichzeitig Sehstörung auf beiden Augen.

Patient, sehr bager und elend, Knochenfisteln. Keine Vergrösserung der Schilddrüse, keine Nagel- und Haaranomalien. Blutbild feht.

Beiderseits ausgesprochener typiseher Keratoconus; Sensibilität auf dem linken Kegel etwas herabgesetzt.

Abderhalden ( $1,5 \mathrm{ccm})$. Ser. -

weil. Pfalz-Düsseldorf.

$$
\begin{aligned}
& "+\text { Thyr. } \\
& "+\text { + Thynus } \\
& "+\text { Nebenniere }++
\end{aligned}
$$

20jâhrige Dame; angeblich sonst gesund, kein Allgemeinbefund. Seit drei Jahren Keratoconus festgestellt; derselbe ist typisch, ohne Hornhauttrübung. Alderhalden (1,5 cem).

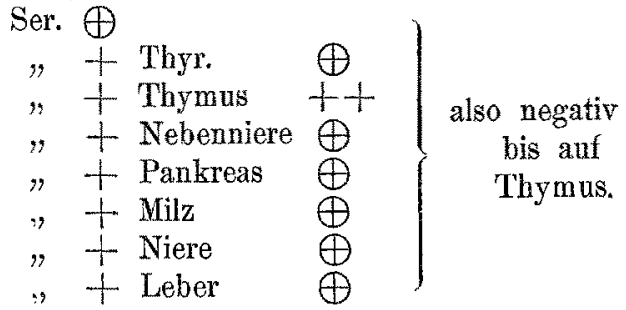

Deutschmann-Hamburg.

18 jähriger junger Mann. Links Keratoconus, während der Beobachtung trat unter Reizerscheinungen Trübung der Spitze des Kegels auf. Rechts normal.

Das linke Auge war 1911 noch normal gefunden worden durch Mannhardt.

"Irgendwelche klinische Basedowerscheinungen bestehen nicht."

Abderhalden $(1,5 \mathrm{cem})$ Ser.

Greeff. 2 Fälle.

$$
\begin{aligned}
& "+\text { Tyr. }- \\
& "+\text { Thymus ++ } \\
& "+\text { Nebenniere } \oplus \\
& " \text { Pankreas }-
\end{aligned}
$$

1. 25 jähriges Fräulein; seit 9 Jahren Abnahme der Sehkraft. Beiderseits ansgesprochener Keratoconus.

Allgemeinbefund fehlt. 
Weitere Untersuch. über Keratoconus mit dom Abderhaldenschen usw. 187

Abderhalden $(1,5 \mathrm{ccm})$.

$\left.\begin{array}{cc}\text { Ser. } \oplus \text { Thyr. } & \oplus \\ "+\text { + Thym. } & ++ \\ "+\text { + Nebenniere } & \oplus \\ "+\text { Pantreas } & \oplus \\ "+\text { Ovar. } & \oplus \\ "+\text { Milz } & \oplus\end{array}\right\}$

also negativ

bis auf

Thymus.

2. 15 jäbriger Junge. Seit Anfang Juni 1913 rapide Abnahme des bis dahin guten Sehvermögens, zugleich Schweilnng der Schilddrüse. Leidet seit der Jugend an starkem Fusschweiss, der im letzten Jahre fast völlig verschwunden ist. Beiderseits hochgradiger Keratoconus. $S=1 / 3$. $^{\circ}$

Abderhalden (1,5 cem).

$\left.\begin{array}{cc}\text { Ser. } \oplus \text { + Thyr. } & \oplus \\ " \quad+\text { Thymus } & ++ \\ "+\text { + Nebenniere } & \oplus \\ "+\text { Pankreas } & \oplus \\ "+\text { Hoden } & \oplus\end{array}\right\} \begin{array}{r}\text { also negativ } \\ \text { bis auf } \\ \text { Thymus. }\end{array}$

Ich habe geglaubt, die Krankengeschichten der verwerteten Fälle, soweit ich sie besitze, mitteilen zu müssen, weil die A b derhaldensche Methodik bei Keratoconus hier zum erstenmal in grösserem Umfang verwendet worden ist und weil ich meine Schlüsse der Kontrolle von anderer Seite zugänglich machen möchte. Zur besseren Übersicht gebe ich zunächst eine Tabelle des Materials, wobei auch die in meiner ersten Arbeit bereits erwähnten Fälle noch einmal mit aufgeführt werden. Aus dem Allgemeinbefund kann ich in der Tabelle natürlich nur die Hauptpunkte hervorheben und verweise im übrigen auf die Krankengeschichten.

Inzwischen sind noch folgende Fälle hinzugekommen:

2 Sera aus der Klinik Axenfeld und eine eigene Beobachtung, die ich zunächst anführe. Frau A. M., 42 Jahre, will seit 6 Jahren ihre Sehstörung in unveränderter Weise haben. R. $-0,75$ cyl. A. $20^{\circ}$ nas. $S=5 / 10$. L. $+1 \mathrm{sph} . \subsetneq-3 \mathrm{cyl}$. A. $35^{\circ}$ nas. $S=5 / 15$. Beiderseits keratoskopisch und skiaskopisch typischer Keratoconus mit absolut klarer Hornhaut.

Patient hat eine ausgesprochene Struma, die früher noch grösser gewesen sein soll, zahlreiche trophische Störungen an den Nägeln, ist sehr frühzeitig ergraut and immer ,nervös“. Interessanterweise behauptet sie, dass ihre Angen viel ,kleiner" geworden seien. Dabei sind die Lidspalten auffallend weit, und man kann von Zeit zu Zeit ein plötzliches Emporsteigen des oberen Lides beobachten, so dass die Sklera oberhalb des Hornhautrandes 
sichtbar wird. Tachykardie besteht nicht, auch keine Anomalien der Schweisssekretion. Die Struma und das Lidphänomen sprechen für forme fruste des Basedow.

Abderhalden. 26. V. 1914. 1,5 cem.

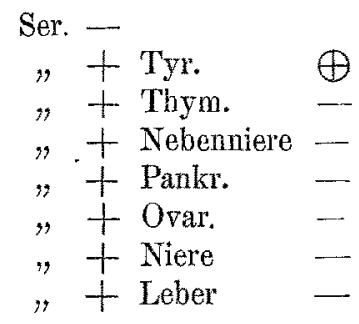

Axenfeld: Karoline Maulbetsch, 26 Jahre.

Sieht seit einigen Jahren in der Nähe und Ferne schlecht. Vor 2 Jahren erhielt sie answärts Brille. Beiderseits $+2 D$ cyl. A. horiz. Damals sah sie mit derselben gut.

Jetzt beiderseits Keratoconus; stärkste Prominenz beiderseits dicht unterhalb der Hornhautmitte, beiderseits zarte Cataracta punctata.

R. ohne Glas $S=6 / 20-6 / 15+4$ eyl. A. horiz. $S=6 / 10 \mathrm{p}$.

L. ohne Glas $S={ }^{6} / 36+4$ cyl. A. $10^{\circ}$. $S=6 / 12$.

Blasse, zarte Frau, von jeher etwas nervös und aufgeregt. Schilddrüse beiderseits palpabel. Am Cor leichtes systolisches Geräusch. Lungen, Abdomen o. B.

Hämogl. $70 \%$. Erythroz. 4800000 L. 7200 , davon neutr. 64, kleine Lymphoz. $28 \%$, grosse Lymploz. $6 \%$, eos. $2 \%$.

Abderhalden $1 \mathrm{cem} .11 .12$. V. 1914.

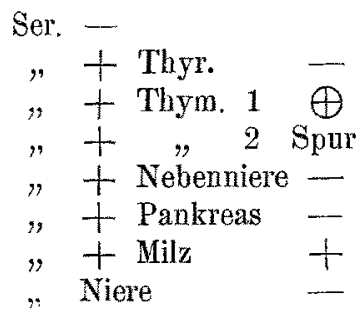

Axenfeld: Fall Hoffmann. weibl.

1910. Rechts ziemlich vorgeschrittener, links eben erkennbarer Keratoconus.

R. -3 cyl. $\pi 30^{\circ} . S=6 / 18^{*}$ L. E. $S=6 / 6^{\circ}$

1913. Steht in dauernder Kontrolle, Behandlung Pilocarpin. Links am Keratoconus keine Veränderung.

28. V. 1913. Interne Untersuchung (Prof. de la Camp). Patientin ist eine typische Sympathicotonica, d. h. vasomotorisch ibererregbare Patientin. Starke Reaktion der Hautgefässe, schneller Wechsel der Füllung der einzelnen Gefässprovinzen. Dysmennorrhoe mit starken Beschwerden. Sehilddrüse palpabel vergrössert. Blut: keine Lymphozytose, $80 \%$ Hämogl., 7900 Leukoz. Röntgenolog.: mitrales erregt schlagendes Herz. Therapie: Pankreontabletten. 
Weitere Untersuch. über Keratoconus mit dem Abderhaldenschen usw. 189

1914. Änderung des Astigmatismus am linken Auge, 2 cyl. $\ngtr 45^{\circ}, S$ $=51_{6}$ bis 5 . Leichte Zunahme des Keratoconus. Patientin befindet sich sonst wohl.

Abderbalden. 1 cem. 11.j12. V. 1914.

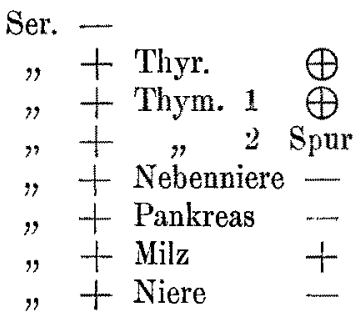

Zu dem Ergebnis der beiden letzten Versuche ist folgendes zu bemerken: die überraschende positive Reaktion mit Milz in beiden Fällen musste zunächst auf einen Versuchsfehler bezogen werden. Zwei mit demselben Organ gleichzeitig untersuchte Sera blieben aber vollkommen negativ, wie ich überhaupt bei sehr zahlreichen Kontrolluntersuchungen mit Milz nur ganz vereinzelt positive Befunde gesehen habe. Da ich auch bei allen seit dem 12. Mai angestellten Versuchen mit dem gleichen Organ nur negative Reaktionen erhalten habe, muss ich das Ergebnis in den beiden Keratoconusfällen für zuverlässig ansehen und die Erklärung desselben dahingestellt lassen. Die Schilddrüse war bei Hoffmann sicher positiv.

Die nicht völlig übereinstimmende Reaktion mit den beiden Thymuspräparaten, welche die Entscheidung, ob die Reaktion positiv oder negativ ist, erschwerte, veranlasste mich, Abderhalden urn eine Kontrolle meiner sämtlichen Thymuspräparate nach allen von $A b d e r h a l d e n$ bisher erprobten Methoden zu bitten. Das Ergebnis war, dass sie einwandfrei sind, die Erfahrung hat aber gezeigt, dass das hier als Thymus 2 bezeiehnete Präparat regelmässig erheblich schwächere Reaktionen ergeben hat als alle andern, es ist also wohl minder geeignet, und man ist berechtigt, die Thymusreaktion als schwach positiv zu bezeichnen.

Eine Tabelle über die vorstehenden Untersuchungen, in welcher die Resultate kurz zusammengestellt waren, habe ich in meinem Vortrag auf dem Kongress für innere Medizin in Wiesbaden ${ }^{x}$ gezeigt und zusammenfassend hervorgehoben, dass mir

1) 21. April 1914 . 


\begin{tabular}{|c|c|c|c|c|c|c|c|}
\hline$N r$. & Autor & Fiall & Ser. & Thyr. & Thym. & Nebenn. & Pankr. \\
\hline 1 & E. v. Hippel & Srauss. 32J. of & - & - & + & - & - \\
\hline 2 & $"$ & $\begin{array}{l}\text { Schultze. } \\
18 \mathrm{~J} . \text { ठ }\end{array}$ & - & $\theta$ ? & +++ & $+t$ & \\
\hline 3 & ", & Singer. 8J. & - & - & $\oplus$ & - & $\oplus$ \\
\hline 4 & $"$ & $\begin{array}{l}\text { Wischeropp } \\
16 \mathrm{~J}, \delta\end{array}$ & - & - & - & - & - \\
\hline 5 & $"$ & $\begin{array}{l}\text { Günther. } \\
25 \% \text { \% }\end{array}$ & - & + & $\oplus$ & - & - \\
\hline 6 & $"$ & $\begin{array}{l}\text { Bräuer. } \\
39 \mathrm{~J} . \text { 옹 }\end{array}$ & - & - & - & & \\
\hline 7 & Fleischer & Fall 1 & - & - & -- & - & - \\
\hline $\begin{array}{l}8 \\
9\end{array}$ & $"$ & Fall 2 & - & + & + & +1 & \\
\hline 9 & $"$ & $\begin{array}{l}\text { Fall } 4 \\
\text { Fall } 5\end{array}$ & - & $\oplus$ & $\oplus$ & $\omega^{(?)}$ & $-(?)$ \\
\hline 11 & $"$ & Fall 6 & $\oplus$ & + & $t+$ & $\oplus(-)$ & \\
\hline 12 &,$"$ & Fall 9 & 岗 & +1 & $+1+1$ & $1+$ & + \\
\hline 13 & $\begin{array}{l}\text { Axenfeld } \\
\text { Augstein }\end{array}$ & $32 \pi .2$ & $\Phi$ & + & +1 & $\theta(-)$ & $\div$ \\
\hline & & Fall 1 & $\oplus$ & + & $\begin{array}{c}+1+ \\
++\end{array}$ & ++ & + \\
\hline 15 & $"$ & $\begin{array}{l}\text { Fall } 2 \\
25 \mathrm{~J} .0\end{array}$ & - & - & - & - & \\
\hline 16 & " & $48 \mathrm{~J}$ & - & $\oplus$ & $\oplus$ & - & - \\
\hline 17 & Siegrist & $\begin{array}{l}\text { Fall } 1 \\
17 \mathrm{~J} . \mathrm{J}\end{array}$ & - & - & $\underline{-}$ & $\oplus$ & \\
\hline 18 & ${ }_{1}^{1} \quad \pi^{\circ}$ & $\begin{array}{l}\text { Fall } 2 \\
16 \text { J. 오 }\end{array}$ & - & $\oplus$ & $\oplus$ & - & - \\
\hline $\begin{array}{l}19 \\
20\end{array}$ & A. v. Hippel & $\begin{array}{l}19 \mathrm{~J} . \text { 옹 } \\
16 \mathrm{~J} .\end{array}$ & $\bar{\oplus}$ & $\overline{1}$ & $\oplus$ & + & + \\
\hline $\begin{array}{l}21 \\
22 \\
02\end{array}$ & $\begin{array}{l}\text { Wagenmann } \\
\text { Scheffels }\end{array}$ & $19 \stackrel{\text { 今े }}{\mathrm{J}}$. & E & $\stackrel{\oplus}{\oplus}$ & $t$ & $\oplus$ & $\bar{\oplus}$ \\
\hline 23 & $n$ & $42 \mathrm{~J}$. 오. & - & $\oplus ?$ & $\oplus$ & - & - \\
\hline 24 & Brenske & 27 J. 오 & - & - & - & - & - \\
\hline $\begin{array}{l}25 \\
26\end{array}$ & $\begin{array}{l}\text { Reis } \\
\text { Koll }\end{array}$ & $\begin{array}{l}\delta \\
\phi\end{array}$ & - & & $\bar{\oplus}$ & $\bar{\oplus}$ & $\bar{t}$ \\
\hline 27 & Engelbrecht & $18 \mathrm{~J}$ б & - & $\oplus$ & - & $+t$ & \\
\hline $\begin{array}{l}28 \\
29\end{array}$ & $\begin{array}{c}\text { Pfalz } \\
\text { Deutschmann }\end{array}$ & $\begin{array}{l}20 \mathrm{~J} . \\
18 \mathrm{~J} . \\
\end{array}$ & $\underline{\oplus}$ & $\mathbb{\oplus ( - )}$ & +1 & $\oplus(-)$ & $\oplus(-)$ \\
\hline $\begin{array}{l}30 \\
31\end{array}$ & $\begin{array}{c}\text { Greeff } \\
\quad "\end{array}$ & $\begin{array}{l}25 \mathrm{~J} .9 \\
15 \mathrm{~J} .+5\end{array}$ & $\oplus$ & $\oplus(一)$ & $+t$ & $\oplus(-)$ & $\oplus(-)$ \\
\hline 32 & E. у. Hippel & $42 \mathrm{~J}$. 오 & - & $\oplus$ & - & - & - \\
\hline 33 & Axenfeld & $26 \mathrm{~J} .9$ & - & $\oplus$ & $\oplus$ & - & - \\
\hline 34 &, & ?오 & - & - & $\oplus$ & - & - \\
\hline
\end{tabular}


Weitere Untersuch. über Keratoconus mit dem Abderhaldenschen usw. 191

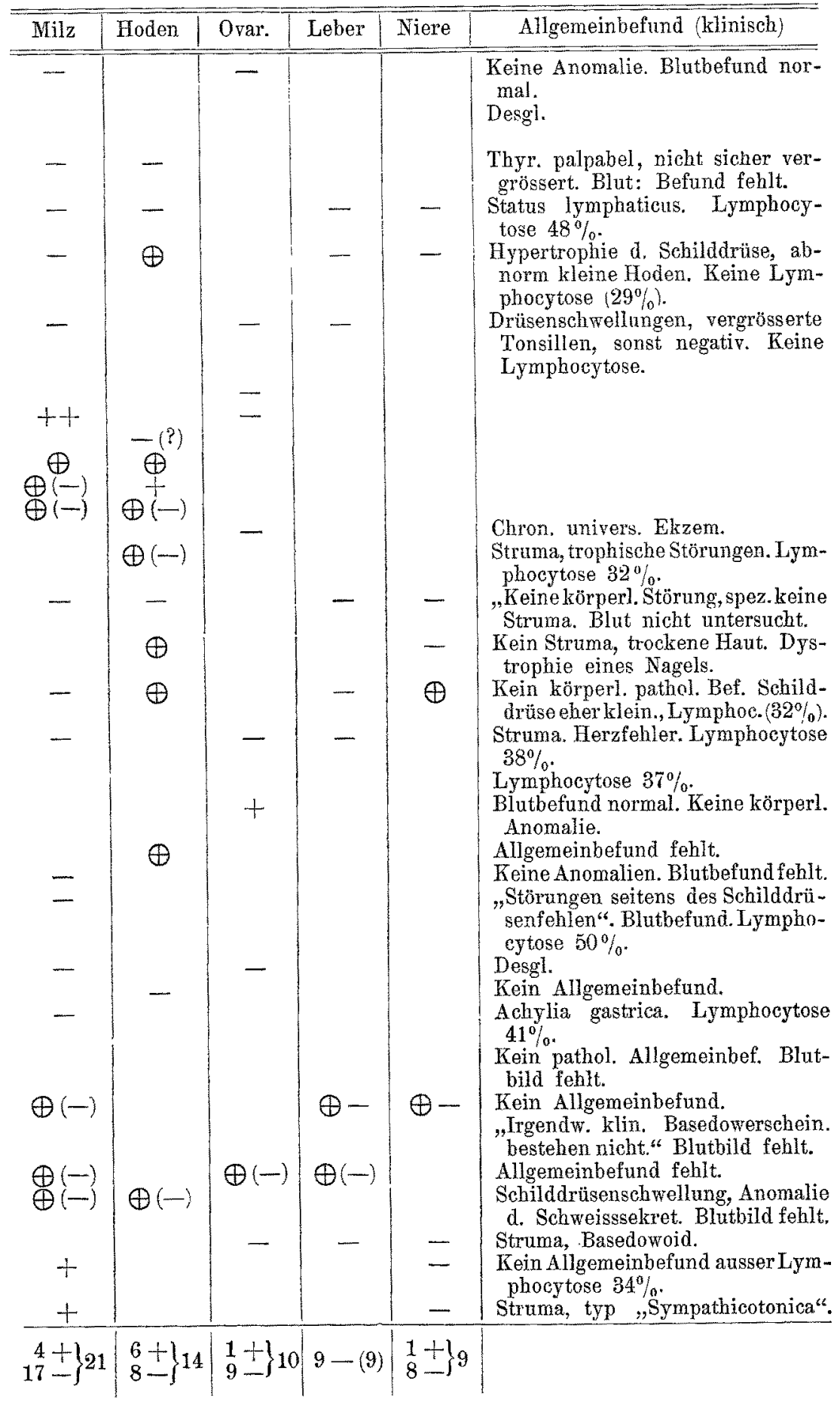


die Ergebnisse die Ansicht zu stützen scheinen, wonach der Keratoconus mit Störungen der inneren Sekretion zusammenhänge, dass aber wohl nicht einfach eine Hypothyreose anzunehmen sei, sondern dass der häufige Abbau mehrerer Organe und die enorme Häufigkeit der Thymusreaktion besondere Beachtung verdiene. Nähere Ausführungen waren unmöglich, da mein Vortrag sich bei einer Redezeit von 10 Minuten wesentlich mit den in der folgenden Arbeit niedergelegten Untersuchungen beschäftigte.

Es ist deshalb an dieser Stelle durchaus erforderlich, meine Resultate mit möglichst scharfer Selbstkritik zu beleuchten.

Wer die Literatur über die Abderhaldenschen Methoden, dio wesentlich in der Münch. med. Wochenschr. 1913 und $1914 \mathrm{zu}$ finden ist, genauer verfolgt hat, der weiss, dass allmählich eine Anzahl möglicher Fehlerquellen aufgefunden ist, die gewisse Verschärfungen der Kontrollen notwendig gemacht haben. Ich habe bei meinen Untersuchungen darauf selbstverständlich stets Rücksicht genommen, ein Teil derselben fällt aber in die Zeit, wo die Vorschriften noch weniger streng waren. Auch jetzt noch behauptet bekanntlich eine Anzahl von Autoren, dass man mit dem Dialysierverfahren überhaupt keine brauchbaren Resultate erhalten könne. Ich selbst bin auf Grund nunmehr ziemlich ausgedehnter Erfahrungen der entgegengesetzten Ansicht, halte es aber auch meinerseits für notwendig, dass man gegebenenfalls in der Lage sein muss, ein bestimmtes Organ mehrmals anzusetzen, zum Einzelversuch zahlreiche Organe zu benutzen, ein Kontrollserum mit den verwendeten Organen zu führen, unter Umständen nach einigen Tagen noch einmal Blut zu entnehmen u. dgl. Wann Situationen eintreten können, wo dies nötig erscheint, lernt man nur bei dem praktischen Arbeiten mit der Methode, es hat deshalb keinen $\mathrm{Zweck}$, hier näher darauf einzugehen.

Ich erwähne diese Dinge nur, weil ich hervorheben muss, dass ich nicht in allen Fällen die allmählich als wünschenswert erkannten Kontrollen durchführen konnte, zum Teil auch deswegen, weil ich von auswärts meistens nur einmal Serum bekam, oft in nicht sehr grosser Menge. Dazu kommt noch die Möglichkeit, dass das Serum durch den Transport leiden könnte, und die manchmal vielleicht nicht zweckmässige Vorbereitung desselben. A b d e r $\mathrm{h}$ alden hat neuerdings darauf hingewiesen, dass schon das $\mathrm{Ab}$ stechen des Blutkuchens Veranlassung zu Hämolyse geben kann; $o b$ das Schütteln beim Transport in den Fällen, wo mir nicht 
Serum, sondern Blut geschickt wurde, etwas schaden kann, weiss ich nicht, halte es aber nicht für ganz unmöglich.

Ich muss deshalb damit rechnen, dass vi elleicht nicht jedes der gewonnenen Resultate von unbedingter Zuverlässigkeit ist. Gebe ich das zu, so liegt der Einwand nahe: wenn ein Resultat nicht stimmt, können alle unzuverlässig sein. Dies ist aber $\mathrm{m}$. E. ausgeschlossen, denn ein Blick auf die Tabelle lehrt schon, in wie ungleicher Häufigkeit die einzelnen Organe abgebaut wurden. Das kann aber nicht auf der eventuell mangelhaften Beschaffenheit einzelner Sera beruhen. Der Beschaffenheit der Organe, die auch zu zahlreichen andern Versuchen benutzt wurden, habe ich stets die grösste Aufmerksamkeit geschenkt. Ich bin deshalb fest überzeugt, dass die Resultate in der Hauptsache zutreffend sind und dass nur ganz vereinzelte Irrtümer vorliegen können. Einer Kontrolle würde z. B. ein Ergebnis wie im Fall 20 mit $5+$ bedürfen. Ich betone noch ausdrücklich, dass die Fälle, welche in jeder Hinsicht vollständig untersucht werden konnten, prinzipiell dieselben Ergebnisse hatten wie die übrigen. Es ist deshalb wesentlich ein Ausdruck der Vorsicht und Gewissenhaftigkeit, wenn ich darauf hinweise, dass mir trotz der grossen Zahl der Versuche weitere Bestätigungen erwünscht sind.

Ich habe mein Material jetzt veröffentlicht, weil ich es für gross genug halte, um daraus begründete Schlüsse zu ziehen, selbst unter der Voraussetzung einzelner Fehler. Ich werde aber die Untersuchungen noch längere Zeit unter Berücksichtigung aller Verbesserungen der Methodik fortsetzen, um zu kontrollieren, ob sich das, was ich bis jetzt gefunden habe, weiter bestätigt. Eigene Fälle, bei denen alle Möglichkeiten der Kontrolle ausgenutzt werden können, werden dabei am wertvollsten sein, ich bin aber auch weiter für jede Unterstützung durch Material dankbar und bitte nur dringend, die geeigneten Massnahmen zu treffen, damit das Serum brauchbar ist: Blutentnahme von $30 \mathrm{ccm}$ mit trockener steriler Nadel in ein weites Gefäss. Abwarten der vollständigen spontanen Gerinnung. Abgiessen des Serums ohne jede Berührung des Blutkuchens. Scharfes Zentrifugieren, bis der Boden des Röhrchens nichts mohr von roter Farbe erkennen lässt. Einsendung des Serums, am besten als Eilpaket im sterilen Gefäss.

Überblicken wir die Tabelle, so finden wir 6 Fälle mit vollkommen negativer Reaktion, 20 mit multiplem Abbau, 1 mit alleinigem Abbau von Schilddrüse und 7 mit alleiniger positiver 
Thymusreaktion. Diese ist überhaupt in der ganzen Serie die weitaus häufigste (25:34), dann folgt Schilddrüse $(17+2(?): 34)$.

Die Kombination von Schildarüse und Thymus bei demselben Fall finden wir 15 mal, wenn wir die zwei fraglichen Thyr. Fälle weglassen.

Die Nebenniere reagiert von den übrigen Organen am häufigsten positiv. Bemerkenswert ist, dass in allen zwölf Fällen, wo multipler Abbau (drei oder mehr Organe) verzeichnet ist, ausnahmslos sowohi Schilddrüse wie Thymus positiv reagieren. Eine Gesetzmässigkeit in dem Hinzukommen anderer positiv reagierender Organe lässt sich nicht auffinden.

Natürlich wäre es von grösstem Interesse, genau angeben zu können, wie oft bei positiver bzw. negativer Reaktion klinisch nachweisbare Anomalien an den in Betracht kommenden Organen oder Abweichungen des Blutbilds vorkommen. In dieser Hinsicht möchte ich auf die Tabelle und die Krankengeschichten verweisen. Aus denselben ergibt sich, dass ein wirklich genauer Allgemeinstatus von völlig sachverständiger Seite in sehr vielen Fällen fehlt, so dass es zwecklos wäre, statistische Angaben machen zu wollen. Scheinbar fehlt eine klinische Erklärung für das Ergebnis der Seroreaktion jedenfalls sehr häufig. Darauf kann ich aber einstweilen aus zwei Gründen keinen besonderen Wert legen. Einmal nämlich wird sich aus der folgenden Arbeit ergeben, dass wirklich zuverlässige Resultate nur dann gewonnen werden, wenn die klinischen Untersuchungen von absolut kundiger Seite nach einheitlichem Plane durchgeführt werden und, wie ich hinzufügen möchte, wenn der Internist eigenes Interesse an der Sache hat und sich die nötige Zeit nimmt, nicht aber dann, wenn solche Fälle in einer überfüllten Poliklinik als lästige Beigabe mit abgemacht werden.

Zweitens haben wir nicht nur mit der Möglichkeit, sondern mit der sicheren Tatsache zu rechnen, dass bei der enormen Feinheit der Abderhalden schen Methode Dysfunktionen einzelner Organe auf diese Weise erkannt werden können, wenn die relativ groben klinischen Methoden noch vollkommen versagen.

Finden wir Übereinstimmung mit der serologischen Untersuchung, so ist das natürlich besonders erfreulich, ein negativer klinischer Befund diskreditiert aber das serologische Ergebnis nicht.

Wie verhalten sich nun die sechs Fälle mit völlig negativer 
Reaktion in klinischer Hinsicht? Müssen wir bei diesen annehmen, dass der Keratoconus nichts mit Störungen der inneren Sekretion. zu tun hat?

In Fall 15 der Tabelle soll der Keratoconus mit Wahrscheinlichkeit angeboren sein; dies würde nicht ausschliessen, dass er auf eine angeborene Anomalie einer (oder mehrerer) Drüse mit innerer Sekretion beruht hat. Diese kann längst kompensiert sein; der negative Ausfall der Reaktion war von vornherein wahrscheinlich. In Fall 7 der Tabelle war der Conus einseitig und bestand seit 8 Jahren, es ist also auch hier wohl möglich, dass die Störungen, die ihn verursachten, längst zur Ausheilung gekommen waren. Das gleiche gilt für Fall 6, während ich über die Fälle 24 und 25 wegen nicht ausreichender klinischer Notizen keine Angaben machen kann. Im Fall 4 ist die negative Reaktion insofern bemerkenswert, als der Keratoconus hier noch nicht sehr lange besteht und sich zurzeit noch deutlich ändert. Ausserdem ist Status lymphaticus und Lymphozytose nachweisbar. Erneute Untersuchung - 4 Monate nach der ersten - hat wieder keinen Abbau eines Organs ergeben - zugleich eine Probe auf die $\mathrm{Zu}$ verlässigkeit der Untersuchung.

Man kann daher einstweilen nur sagen: in der Mehrzahl der Fälle sind mit dem Abderhaldenschen Verfahren Störungen der inneren Sekretion nachzuweisen, es gibt aber auch solche, wo die Reaktion durchaus negativ ist und auch die klinische Untersuchung versagt.

Diese letztere Tatsache schliesst natürlich nicht aus, dass zu der Zeit, wo der Keratoconus entstand, eine Störung der inneren Sekretion vorhanden war, ebenso ist mit der Möglichkeit zu rechnen, dass bei geringfügigen Anomalien Abwehrfermente im Serum zeitweise fehlen können.

Es würde sich also empfehlen, negativ reagierende Fälle in gewissen Abständen nachzuuntersuchen, dann wird sich diese Frage entscheiden lassen.

Vorläufig kann jedenfalls nicht behauptet werden, dass der Nachweis einer Störung der inneren Sekretion regelmässig gelungen sei.

Zur Prüfung der Frage, ob man berechtigt oder genötigt ist, zwei ätiologisch ganz verschiedene Formen von Keratoconus anzunehmen, von denen die eine auf angeborener Anlage, die andere 
auf erworbener Ernährungsstörung beruht, war mein Material wenig geeignet.

Ausser dem bereits erwähnten Fall 15 ist nur noch Nr. 3 zu nennen, bei welchem die Annahme, dass der Keratoconus auf kongenitaler Anlage beruhe, besonders gestützt erscheinen würde. Trotzdem liegt es mir durchaus fern, die Möglichkeit, dass dies öfters der Fall sein könnte, zu bestreiten. Nur würden doch auch hier ähnliche Beziehungen in Betracht kommen, wie zwischen dem angeborenen und dem später entstandenen Schichtstar, d.h. in beiden Fällen könnte in einer Störung der inneren Sekretion die. gemeinsame Ursache liegen. (Vgl. hierzu meine Ausführungen in Schwalbes Handbuch der Missbildungen, S. 55.)

Man wird gut tur, in diesen Dingen keine Dogmen aufzustellen, sondern vorurteilslos allen Möglichkeiten Rechnung zu tragen und in erster Linie für ein genau untersuchtes Tatsachenmaterial zu sorgen.

Wenn man mit dem Abderhaldenschen Verfahren Dysfunktion irgendwelcher Art nachweist, so ist damit zunächst noch keineswegs bewiesen, dass dieselbe in ursächlicher Beziehung zu der Erkrankung steht, die uns zu der Untersuchung veranlasst hat.

Erhält man aber positive Reaktionen an bestimmten Organen bei ein und derselben Krankheit in a uffallender Häufigkeit oder regelmässig, so wird der Zusammenhang schon erheblich wahrscheinlicher, besonders wenn bereitsklinische Erwägungen anf die störung der inneren Sekretion hinwiesen und wenn gar eine organtherapeutische Behandlung einwandfreie Erfolge aufzuweisen hat. In letzterer Beziehung liegen beim Keratoconus noch sehr wenige Erfahrungen vor (Dor, Augstein, Tamascheff). Ich selber kann leider nichts dazu beitragen, da der einzige einigermassen aussichtsvolle Fall Singer wegen der Verständnislosigkeit der Eltern nicht zu regelmässiger Beobachtung und Behandlung gebracht wurde. Im Falle $W$ ischeropp habe ich eine Organtherapie nicht versucht, weil die Serumreaktion negativ war. Es ist also durchaus notwendig, erst weitere Erfąhrungen auf diesem. Gebiet zu sammeln.

Bei den sehr engen funktionellen Beziehungen, in welchen die beiden am häufigsten positiv reagierenden Organe, Schilddrüse und Thymus, stehen, ist mit der Möglichkeit zu rechnen, dass eine 
Weitere Untersuch. über Keratoconus mit dem Abderhaldenschen usw. 197

Thyreoidinbehandlung auch eine Dysfunktion der Thymusdrüse beeinflussen könnte. In der folgenden Arbeit wird davon noch zu sprechen sein.

Soweit die im vorstehenden erörterten Tatsachen ein Urteil erlauben, hat sich der Gedanke von Si egrist, den Keratoconus mit Störungen der inneren 'Sekretion in Zusammenhang zu bringen, als fruchtbar erwiesen und ist durch die Untersuchungen mit der Abder haldenschen Methode wesentlich gestützt worden. Die Ansicht, dass es sich um eine Hypothyreose handle, ist nach dem Ergebnis der Seroreaktion einzuschränken, und wie schon Eingangs erwähnt, ist auf den multiplen Abbau und die überaus häufige Beteiligung der Thymus besonderer Wert zu legen.

Eine Fortsetzung der Untersuchungen unter Ausnutzung aller Verbesserungen der Methodik halte ich für nötig; die Frage nach der Ätiologie ist durch meine Untersuchungen gefördert, aber noch nicht erledigt, besonders da wohl noch für längere Zeit von der Feststellung der Tatsache einer gestörten inneren Sekretion bis zu der Erkenntnis, in welcher Weise diese die merkwürdige Hornhautveränderung hervorruft, ein ziemlich weiter Weg sein wird.

Soweit die Literatur über die Abderhald en sche Methodik für die besprochene Frage von Bedeutung ist, wird auf dieselbe in der folgenden Abhandlung eingegangen werden. 